\title{
Intraoral Scanning with Rubber Dam Isolation in Place for Fabrication of a Chairside Computer-assisted Design and Computer-assisted Manufacture Ceramic Restoration
}

\author{
Carlos A Jurado ${ }^{1}$, Rachel Lederman², Jeffrey Cohen ${ }^{3}$, Akimasa Tsujimoto $^{4}$
}

\begin{abstract}
Aim: The clinical report describes how chairside intraoral scanning can be performed while a rubber dam is in place prior to fabrication of a computer-assisted design and computer-assisted manufacture (CAD/CAM) ceramic restoration.

Background: Recently, combining a chairside CAD/CAM system with rubber dam isolation in place could be used in order to both speed up the restorative process and avoid any contamination to abutment surface, which are the positive effects to patients and clinicians. Unfortunately, manufacturers do not provide guidance on how to make use of chairside CAD/CAM restorations while rubber dam isolation is in place.

Case description: The chief complaint of necessitating a crown after endodontic therapy and the patient was digital restorative procedure in single visit. Intraoral scanning with rubber dam isolation in place was planned and successfully completed in order to fabricate a chairside CAD/ CAM ceramic crown. The software was able to interpose the scan of the prepped tooth with rubber dam isolation into a scan made without the rubber dam from which the tooth had been erased.

Conclusion: Chairside CAD/CAM system can be used to scan, design, and fabricate crowns while rubber dam is kept intraorally. Initial scan without rubber dam is needed in order to interpose the second scan with rubber dam in place.

Clinical significance: Combining the advantages of intraoral scanning and rubber dam isolation in place for the fabrication of a chairside CAD/ CAM ceramic crown in a single visit is feasible.

Keywords: CAD/CAM, Digital imaging, Restoration.

The Journal of Contemporary Dental Practice (2021): 10.5005/jp-journals-10024-3174
\end{abstract}

\section{BACKGROUND}

CAD/CAM in dentistry was first announced in the early $1980 s^{1}$ and the initial technology was limited to two dimensions, and thus it was only able to manufacture inlays. ${ }^{2}$ The first crown was fabricated using the same two-dimensional design capability in 1994, and the software introduced three-dimensional design that allowed it to produce larger restorations, up to three or four fixed unit fixed dental restorations, in $2003 .^{3}$ The updated software provides several tools to successfully design and fabricate advanced complex restorations, such as implant restorations, esthetic veneers, and full mouth reconstructions, in a shorter time than conventional methods. ${ }^{4}$

Chairside CAD/CAM systems in dentistry allow practitioners to manufacture final restorations in a single visit. ${ }^{5}$ Optical images acquired directly from the mouth with intraoral scanners eliminate the need for conventional methods such as polyvinyl siloxane impressions and improve patients' comfort. ${ }^{6}$ Single-visit final restorations eliminate the step of fabricating provisional prosthesis and reduce contamination of the abutments. ${ }^{7}$ In addition, conventional problems with inaccuracy caused by traditional laboratory procedures are diminished with the CAD/CAM system and the milling of the restorations provides more optimum and reliable results. ${ }^{8}$ The accuracy of chairside CAD/CAM technology has been demonstrated, and digital impressions for restorations show similar or even better results than conventional impression techniques. ${ }^{9}$ Furthermore, studies have investigated the marginal and internal fit of restorations made by chairside CAD/CAM system and the results showed clinically acceptable ranges. ${ }^{10,11}$
${ }^{1}$ Texas Tech University Health Sciences Center, Woody L. Hunt School of Dental Medicine, El Paso, Texas, United States of America

${ }^{2,3}$ AT Still University Arizona School of Dentistry and Oral Health, Mesa, Arizona, United States of America

${ }^{4}$ Department of Operative Dentistry, University of lowa College of Dentistry, lowa, United States of America

Corresponding Author: Akimasa Tsujimoto, Department of Operative Dentistry, University lowa College of Dentistry, lowa, United States of America, e-mail: akimasa-tsujimoto@uiowa.edu

How to cite this article: Jurado CA, Lederman R, Cohen J, et al. Intraoral Scanning with Rubber Dam Isolation in Place for Fabrication of a Chairside Computer-assisted Design and Computer-assisted Manufacture Ceramic Restoration. J Contemp Dent Pract 2021;22(8):943-946.

Source of support: Nil

Conflict of interest: None

The use of a rubber dam isolation for restorative procedures may be debatable, but it provides several advantages to the patient and clinician. ${ }^{12}$ Studies have shown that the use of a rubber dam may lead to a higher clinical success rate of restorations in comparison with use of cotton roll isolation..$^{12,13}$ Moreover, it has been demonstrated that the rubber dam isolation reduced microbial aerosols by $70 \%$ and thus the risk of cross-infection. ${ }^{14}$ The placement of a rubber dam may also prevent the aspiration of foreign objects through patient's mouth, which is also important for safety reasons. It can also improve the field of view since the clinician does not need to keep retracting the cheek, lips, and tongue in order to work in the area. ${ }^{15}$ 
Recently, combining a chairside CAD/CAM system for production of restorations with the placement of a rubber dam has become a routine process because it both speeds up the fabrication of the restoration and offers the positive results of isolation with rubber dam. Unfortunately, manufacturers do not provide guidance on how to make use of intraoral scanning while rubber dam isolation is in place. Therefore, the purpose of this clinical report is to describe how to complete whole chairside CAD/CAM ceramic restorative process with a rubber dam in place using digital techniques.

\section{Case Description}

A 40-year-old female patient visited to the AT Still University Arizona School of Dentistry, Arizona, United States, with the chief complaint of necessitating a crown after endodontic therapy. The patient completed endodontic therapy on her maxillary left second premolar two weeks ago (Fig. 1). The patient was informed of the treatment options of having the crown created with digital technology in a single appointment or with the conventional technique in two visits. The patient elected to use a chairside CAD/ CAM restorative procedure in a single visit. The patient's occlusion and esthetic demands were evaluated, and lithium disilicate ceramic block (IPS e.max CAD MT C14, Ivoclar Vivadent, Schaan, Lichtenstein) was selected as the restorative material.

A chairside intraoral scan (Primescan, Dentsply Sirona, Charlotte, North Carolina, United States) was initially conducted on both arches and a buccal bite registration was completed without a rubber dam (Fig. 2). Tooth \#13 was digitally removed using the cut tool (Fig. 3). Rubber dam (Dental Dam, Frontier Dental Supply) isolation was provided from \#10-14, and the dam was held with a clamp (\#5-winged molar, Hu-Friedy, Chicago, Illinois, United States) on tooth \#14. Resin composite fillings were removed and core build-up was conducted. An all-ceramic tooth preparation for $1.5 \mathrm{~mm}$ occlusal reduction and $1.0 \mathrm{~mm}$ deep chamfer margin was provided for tooth \#13, with the distal margin going 1.0 subgingival until no core build-up material was
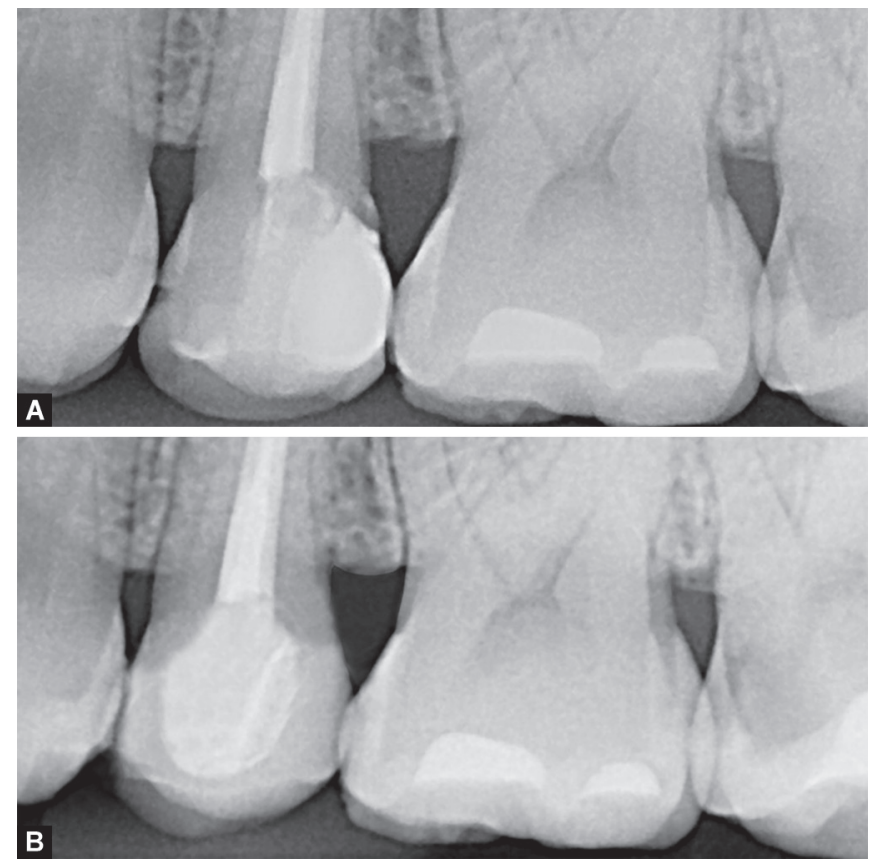

Figs $1 \mathrm{~A}$ and B: (A) Pre- and (B) Postoperative bitewing X-ray images present on the margin. A new intraoral scan with the rubber dam in place, this time of only the occlusal surface of teeth \#12 and \#14 and the entire prepped tooth \#13, was performed, and the image was automatically interposed in the previous image of the teeth without the rubber dam (Fig. 3). A CAD/CAM lithium disilicate block was digitally designed (CEREC 5.1, Dentsply Sirona) and milled (CEREC MC XL, Dentsply Sirona) (Fig. 4). The restoration was sintered (Programat CS2, Ivoclar Vivadent) following the manufacturer's recommendation.

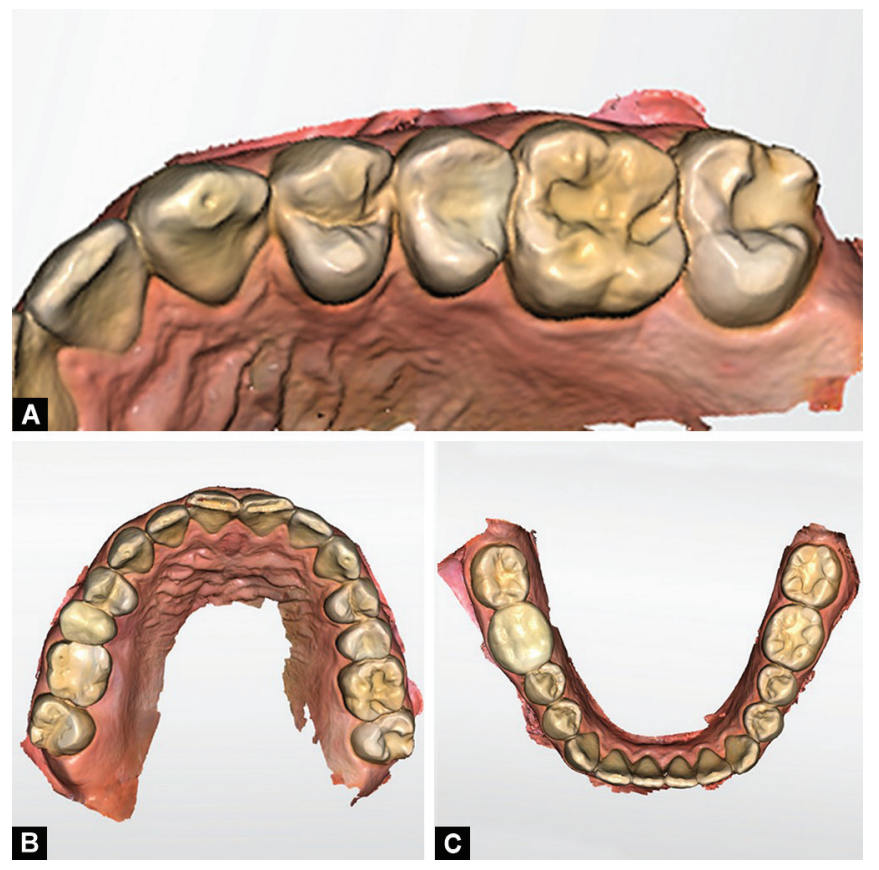

Figs $2 A$ to $C$ : Preoperative intraoral views. (A) Initial scanning for upper right teeth; $(B, C)$ Both as maxillary and mandibular arches
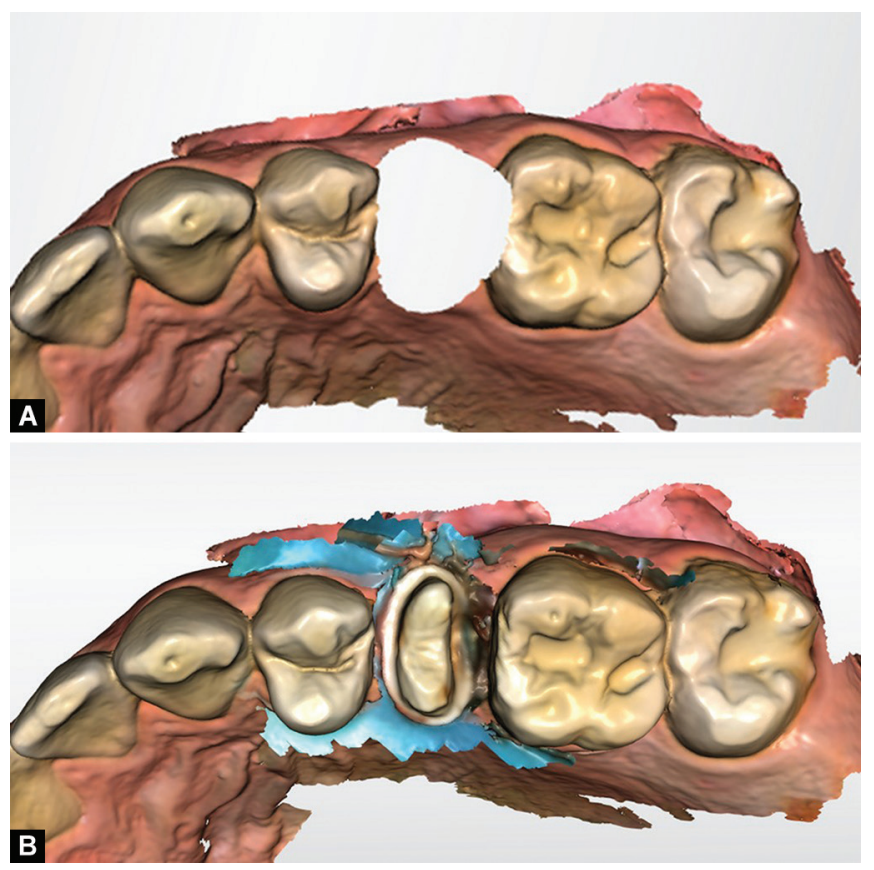

Figs $3 A$ and $B$ : (A) Initial scanning for digital removal and (B) Merging second scan for abutment of tooth \#13 
Cementation was conducted while the rubber dam isolation was still in place, according to the following procedure (Fig. 6). The tooth surface was phosphoric acid etched, rinsed, and air dried with three-way syringe, and treated using primer (Primer A $+B$, Multilink Autolink, Ivoclar Vivadent) for 20 seconds. The restorative surface was etched with $4.9 \%$ hydrofluoric acid (IPS Porcelain Etch, Ivoclar Vivadent), and rinsed with water and air dried, and treated with primer (Monobond Plus, Ivoclar Vivadent) for 20 seconds. The restoration was cemented with conventional resin luting cement (Multilink Automix, Ivoclar Vivadent), and excess cement was removed with micro brushes and an explorer; initial tack-curing was completed on buccal and lingual surfaces for 3 seconds each, then glycerin gel was applied on the margins and final curing was provide on occlusal and buccal surfaces for 10 seconds each. Excess cement was removed again with an explorer and superfloss. The restoration was polished with a ceramic polishing kit (e.max Finishing/Polishing, Brasseler, Savannah, Georgia, United States). The rubber dam was removed, ruminants of excess cements at the marginal area were checked, occlusion was evaluated, and no modifications were needed (Fig. 5). At the 1 year follow-up, patient was still satisfied for the restoration with shortened treatment time and good results.

\section{Discussion}

The presented clinical workflow successfully achieved the fabrication of a lithium disilicate crown using a chairside CAD/CAM system in a single appointment with the rubber dam isolation in place for the entire process (Fig. 6). Combining the advantages of intraoral scanning and rubber dam for fabrication of restorations in a single visit speeds up the process of the intraoral scanning, design, and cementation, and also ensures optimal isolation for bonding protocols. An initial scan without the rubber dam is needed prior to placing the rubber dam. This initial scan provides

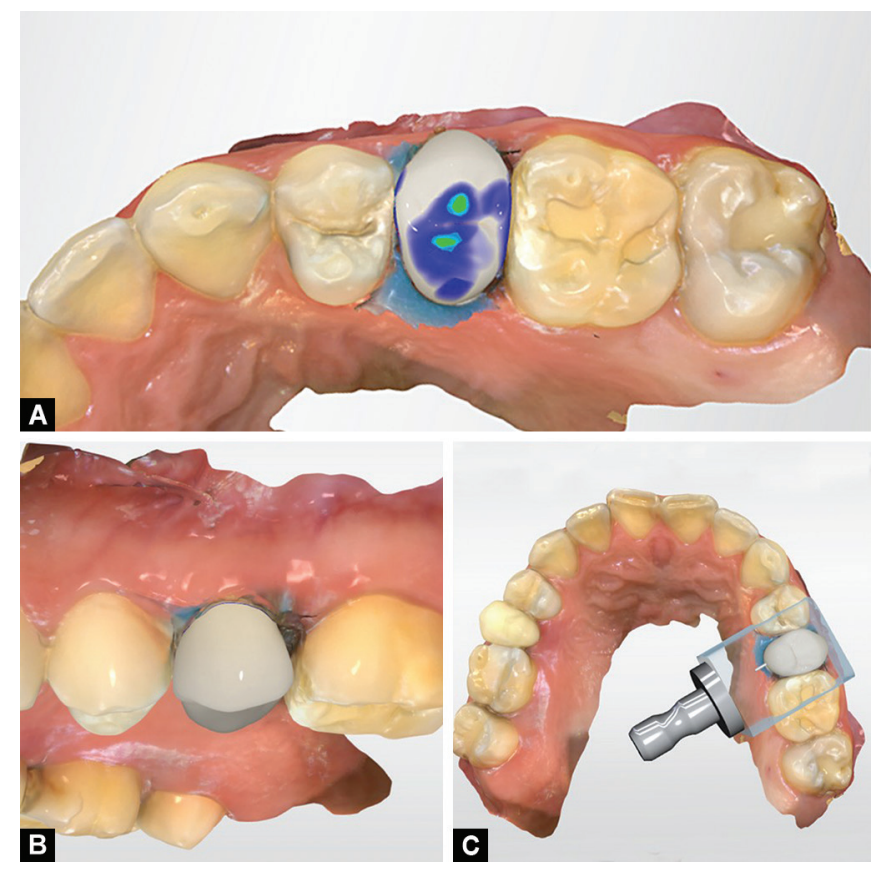

Figs 4A to $C$ : $(A)$ Designing CAD/CAM crown, the rubber dam; $(B, C)$ Designed buccul view and planned CAD/CAM block set-up for milling process of the lithium disilicate crown guidance for stitching in the second scan, and the software can superimpose the two images. In this case, the crown preparation after core build-up was performed subgingivally until sound tooth structure on the margin was found. A new scan of the final tooth preparation was conducted with the rubber dam isolation in place, and this scan only captured the occlusal surface of the posterior tooth until the software recognized the previous scan, at which point the prepped tooth was fully scanned. Since the second image started on the occlusal surface of the adjacent tooth, the software was able to recognize the previous scan and the new image of the prepped tooth successfully replaced the empty area. Thus, multiple tooth isolation, of at least three units, is required. Once the software recognizes the area, the clinician should move the camera to the prepped tooth and scan all of its surfaces. The new image of the prepped tooth with rubber dam was interposed in the previous image and filled the area that was previously cut. Once the prepped tooth has been digitally captured, then all the following steps could be achieved while retaining the rubber dam isolation in position.

Early chairside CAD/CAM systems had many limitations and drawbacks. The initial CEREC software only allowed the clinician to fabricate inlays and it took almost 15 years to gain the ability to fabricate single crowns, and more than two decades to progress from two to three dimensional images. ${ }^{1}$ Clinicians currently have many options when selecting use with these systems. The chairside CAD/CAM system used in this clinical report was Primescan (Dentsply Sirona), which is one of the newer apparatus of this type of technology. According to the manufacturer, the software has been improved and gives the opportunity to scan up to $20 \mathrm{~mm}$ depth, taking more than 50,000 images per second and process more than 1,000,000 3D points per second. ${ }^{16}$ However, further studies are needed to evaluate apparatus from other CAD/CAM systems that have also developed intraoral scanners with advanced technology and novel features.
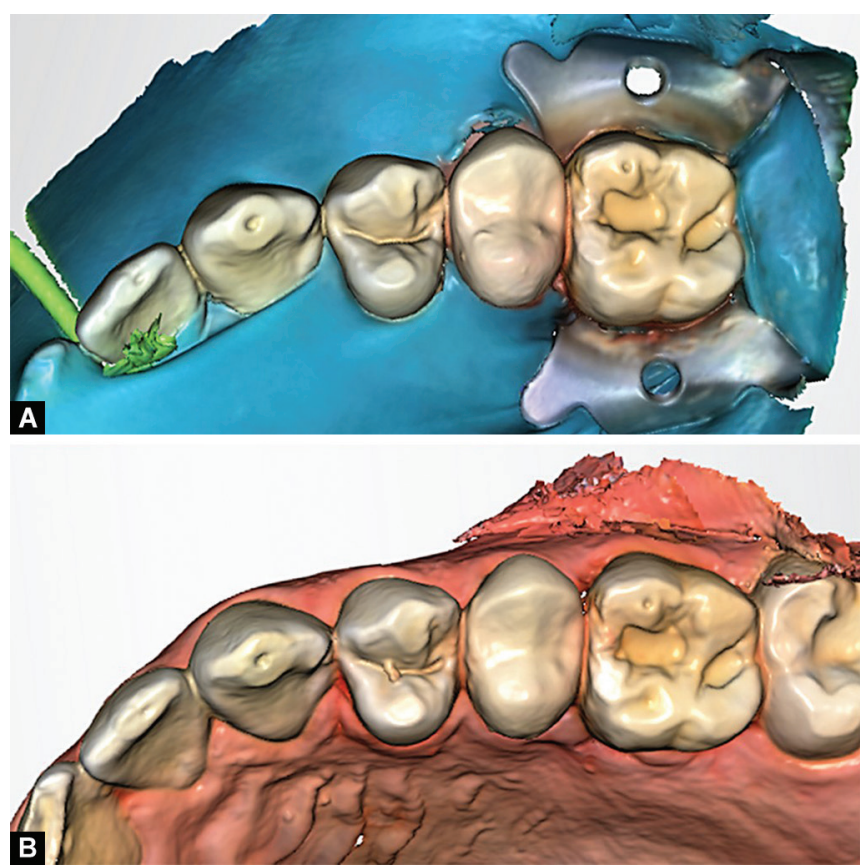

Figs $5 \mathrm{~A}$ and $\mathrm{B}$ : (A) Maintaining the rubber dam during the cementation process and (B) Final prosthesis 


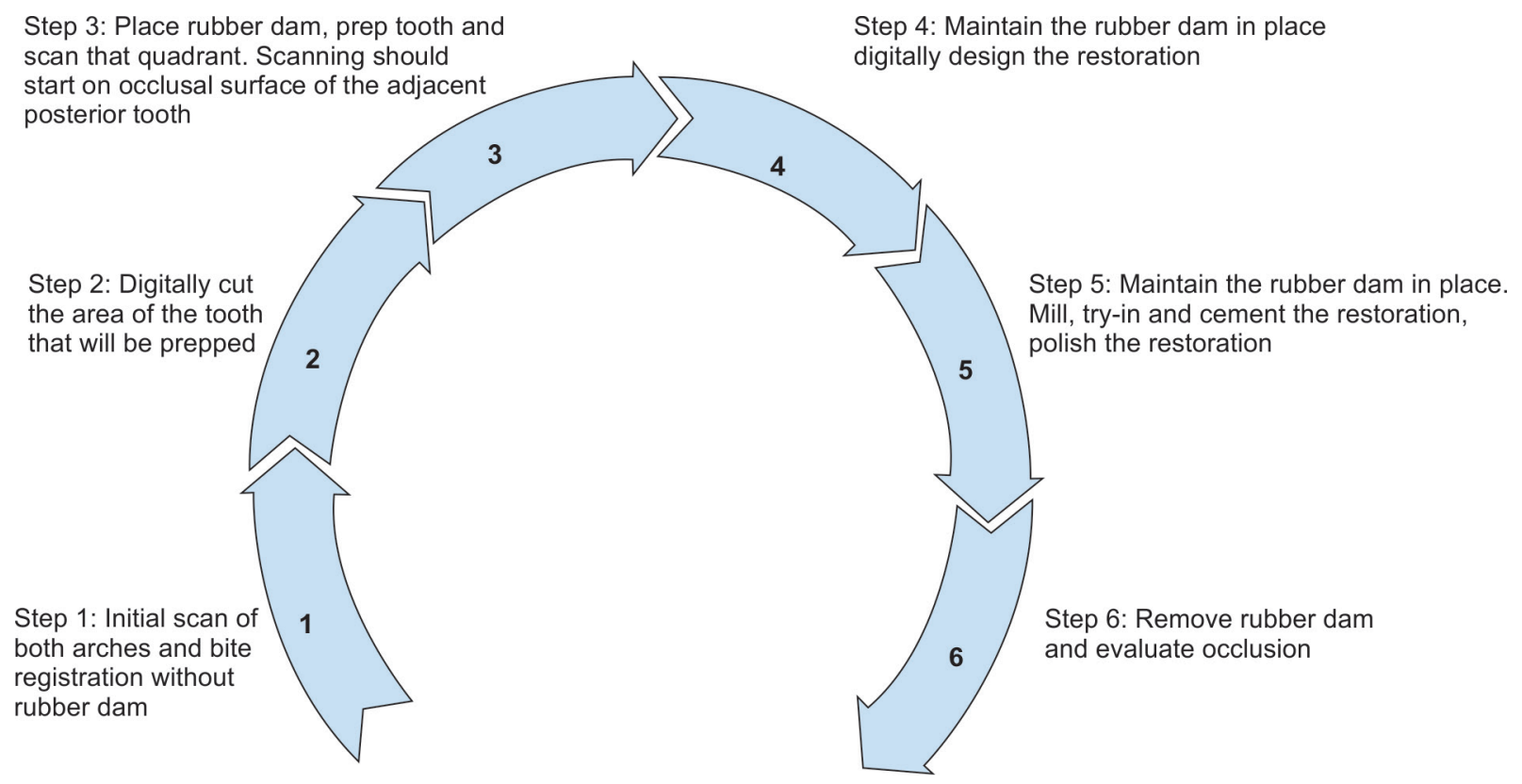

Fig. 6: Clinical workflow for the fabrication of a chairside CAD/CAM lithium disilicate restoration in a single visit with the rubber dam in place

This simple workflow reduced the appointment time because there was no need to remove the rubber dam isolation for intraoral scanning purposes and place new rubber dam isolation prior to cementation. In addition, the risk of saliva and breathing contamination of the abutment surface during intraoral scanning can be minimized. Although this protocol was successful for a single restoration, further studies should evaluate the scanning and fabrication of multiple restorations at the same time.

\section{Conclusion}

Combining the advantages of intraoral scanning and rubber dam isolation for the fabrication of a lithium disilicate ceramic crown in a single appointment is feasible. All the steps necessary for single-visit chairside CAD/CAM restorations can be achieved with rubber dam isolation in place. Intraoral scanning can be performed with rubber dam isolation in place, but an initial scan without rubber dam isolation is needed in order to interpose the final image with the rubber dam.

\section{References}

1. Blatz MB, Conejo J. The current state of chairside digital dentistry and materials. Dent Clin North Am 2019;63(2):175-197. DOI: 10.1016/j. cden.2018.11.002.

2. Mörmann WH, Bindl A. All-ceramic, chairside computer-aided design/computer-aided machining restorations. Dent Clin North Am 2002;46(2):405-426. DOI: 10.1016/s0011-8532(01)00007-6.

3. Reich SM, Wichmann M, Rinne H, et al. Clinical performance of large, all-ceramic CAD/CAM-generated restorations after three years: a pilot study. J Am Dent Assoc 2004;135(5):605-612. DOI: 10.14219/ jada.archive.2004.0248.

4. Jurado CA, Tsujimoto $A$, Watanabe $H$, et al. Chairside CAD/CAM fabrication of a single-retainer resin bonded fixed dental prosthesis: a case report. Restor Dent Endod 2020;45(2):e15. DOI: 10.5395/ rde.2020.45.e15.
5. Miyazaki T, Hotta Y, Kunii J, et al. A review of dental CAD/CAM: current status and future perspectives from 20 years of experience. Dent Mater J 2009;28(1):44-56. DOI: 10.4012/dmj.28.44.

6. Shimizu S, Shinya A, Kuroda S, et al. The accuracy of the CAD system using intraoral and extraoral scanners for designing of fixed dental prostheses. Dent Mater J 2017;36(4):402-407. DOI: 10.4012/dmj.2016-326.

7. Gold D, Hahnel SF, Schierz O, et al. Practicability of a chairside approach for characterizing CAD/CAM resin-based composites. J Oral Sci 2020;62(4):430-434. DOI: 10.2334/josnusd.19-0507.

8. Memari Y, Mohajerfar M, Armin A, et al. Marginal adaptation of CAD/ CAM all-ceramic crowns made by different impression methods: a literature review. J Prosthodont 2019;28(2):e536-e544. DOI: 10.1111/ jopr.12800.

9. Seelbach P, Brueckel C, Wöstmann B. Accuracy of digital and conventional impression techniques and workflow. Clin Oral Investig 2013;17(7):1759-1764. DOI: 10.1007/s00784-012-0864-4.

10. Nakamura T, Dei N, Kojima T, et al. Marginal and internal fit of Cerec 3 CAD/CAM all-ceramic crowns. Int J Prosthodont 2003;16(3):244-248. PMID: 12854786.

11. Lee KB, Park CW, Kim KH, et al. Marginal and internal fit of all-ceramic crowns fabricated with two different CAD/CAM systems. Dent Mater J 2008;27(3):422-426. DOI: 10.4012/dmj.27.422.

12. Wang $Y, \mathrm{Li}$ C, Yuan $\mathrm{H}$, et al. Rubber dam isolation for restorative treatment in dental patients. Cochrane Database Syst Rev 2016;9:CD009858. DOI: 10.1002/14651858.CD009858.pub2.

13. Keys W, Carson SJ. Rubber dam may increase the survival time of dental restorations. Evid Based Dent 2017;18(1):19-20. DOI: 10.1038/ sj.ebd.6401221.

14. Villani FA, Aiuto R, Paglia L, et al. COVID-19 and dentistry: Prevention in dental practice, a literature review. Int J Environ Res Public Health 2020;17(12):4609. DOI: 10.3390/ijerph17124609.

15. Imbery TA, Carrico CK. Dental dam utilization by dentists in an intramural faculty practice. Clin Exp Dent Res 2019;5(4):365-376. DOI: 10.1002/cre2.191.

16. Skramstad MJ. Welcome to Cerec Primescan AC. J Comput Dent 2019;22(1):69-78. PMID: 30848256. 